Mens

revue d'histoire intellectuelle de l'Amérique française

\title{
La (non-)réception critique de Céline et de Drieu la Rochelle dans les périodiques québécois des années 1930 : ignorance ou esprit chouan?
}

\section{Sébastien Côté}

Volume 4, numéro 1, automne 2003

URI : https://id.erudit.org/iderudit/1024627ar

DOI : https://doi.org/10.7202/1024627ar

Aller au sommaire du numéro

Éditeur(s)

Centre de recherche en civilisation canadienne-française

ISSN

1492-8647 (imprimé)

1927-9299 (numérique)

Découvrir la revue

Citer cet article

Côté, S. (2003). La (non-)réception critique de Céline et de Drieu la Rochelle dans les périodiques québécois des années 1930 : ignorance ou esprit chouan? Mens, 4(1), 39-67. https://doi.org/10.7202/1024627ar
Résumé de l'article

Nous cherchons ici les raisons du quasi-silence des intellectuels québécois des années 1930 autour de Céline et Drieu la Rochelle, des auteurs qui faisaient pourtant partie du champ littéraire, à tout le moins par l'intermédiaire des périodiques français. Dans ce paysage littéraire plutôt conservateur, les lectures de Berthelot Brunet et Valdombre surprennent parfois, ce qui montre que certaines œuvres « dissonantes » circulaient malgré tout et qu'on pouvait en parler dans la presse. Mais d'autres noms ne resurgirent que bien plus tard. Nous croyons que cette discrétion était surtout stratégique, car un roman diffusé confidentiellement passe plus facilement inaperçu des autorités. 


\title{
LA (NON-)RÉCEPTION CRITIQUE DE CÉLINE ET DE DRIEU LA ROCHELLE DANS LES PÉRIODIQUES QUÉBÉCOIS DES ANNÉES 1930 : IGNORANCE OU ESPRIT CHOUAN ? 1
}

\author{
Sébastien Côté \\ Département de littérature comparée \\ Université de Montréal
}

\section{RÉSUMÉ}

Nous cherchons ici les raisons du quasi-silence des intellectuels québécois des années 1930 autour de Céline et Drieu la Rochelle, des auteurs qui faisaient pourtant partie du champ littéraire, à tout le moins par l'intermédiaire des périodiques français. Dans ce paysage littéraire plutôt conservateur, les lectures de Berthelot Brunet et Valdombre surprennent parfois, ce qui montre que certaines œuvres " dissonantes » circulaient malgré tout et qu'on pouvait en parler dans la presse. Mais d'autres noms ne resurgirent que bien plus tard. Nous croyons que cette discrétion était surtout stratégique, car un roman diffusé confidentiellement passe plus facilement inaperçu des autorités.

\section{ABSTRACT}

This article examines the near silence of Quebec intellectuals in the 1930s regarding the work. of French writers Céline and Drieu la Rochelle, whose novels were 
nonetheless part of the literary landscape, at least through the intermediate of French periodicals. In spite of French Canada's essentially conservative literary culture, some books read by Berthelot Brunet and Valdombre could be quite surprising, which shows that some "discordant" works did circulate, and that they could be discussed in the press. However, many authors would only appear much later in Quebec's literary landscape. We believe this discretion was mainly strategic, as a novel with a limited distribution was more likely to remain unnoticed by the authorities.

À l'occasion du quatrième centenaire de la découverte du Canada, plusieurs ouvrages retiennent l'attention des critiques français, dont le Jacques Cartier de Charles de la Roncière, Le Canada, les deux races d'André Siegfried, Le Français au Canada de Lionel Groulx et l'Histoire du Canada de Jean Bruchési. Cette commémoration constitue un indicateur important des idées ambiantes (un "nœud idéologique ", selon l'expression de Philippe $\mathrm{Hamon}^{2}$ ) en ce qu'elle cristallise en quelques textes le discours tenu par la classe intellectuelle française sur son ancienne colonie. Un article du critique parisien Octave Aubry, d'abord paru dans l'hebdomadaire de droite Candide, puis reproduit dans L'Ordre (quotidien d'Olivar Asselin) du 24 janvier 1935, constitue à cet égard un exemple des plus représentatifs. L'auteur y rend compte de divers ouvrages récents portant sur le Canada, non sans recourir à une entrée en matière plutôt larmoyante:

Ce n'est pas un sujet dont un Français puisse parler sans un frémissement du cœur. Sans doute n'y a-t-il pas une de nos provinces, aujourd'hui encore, qui nous soit plus chère que le Canada, la première née de nos colonies [...]. Les Canadiens sont demeurés nos frères et nos garants. Ils ont même, hélas !, gardé nos traditions mieux que nous. Nous éprouvons pour eux de la tendresse et du remords ${ }^{3}$. 
Aubry reprend même à son compte le style mythifiant du courant historiographique alors en vogue, une méthode positiviste encore fortement inspirée par la psychologie des peuples et à laquelle Lionel Groulx et Jean Bruchési souscrivent. Ainsi se succèdent dans son compte rendu les mots " épopée ", "vertus de notre sang ", «le miracle canadien », «fidélité franche et modeste », «la petite colonie de jadis, leur victime ", "ces éléments français ne s'assimilent pas", comme si l'auteur avait voulu poursuivre la métaphore filée amorcée par les historiens qu'il venait de fréquenter. Cette émotion est partagée par l'académicien Henry Bordeaux, écrivain catholique et figure omniprésente dans le champ littéraire du Québec de l'entre-deux-guerres. Dans Nouvelle et vieille France, livre de circonstance publié à la suite de sa visite officielle au Canada français en 1934, Bordeaux évoque Jacques Cartier et les coureurs des bois, avant de railler la célèbre boutade de Voltaire. Au cours de ce voyage effectué au nom de l'Académie française, le plus beau reliquat de l'Ancien Régime, il prononce devant les dignitaires de l'Université Laval un discours particulièrement flatteur, sans pour autant s'éloigner de la rhétorique habituelle: "Je vous apporte en son nom l'hommage de trois cents ans de langue et de culture françaises, à vous qui avez sauvé cette langue dans le Nouveau Monde et qui, avec elle, avez gardé notre foi et nos traditions ${ }^{4} »$.

Étrangement, Louis-Ferdinand Céline et Pierre Drieu la Rochelle, deux écrivains infiniment moins épris de conventions que Bordeaux, reprennent ces lieux communs séculaires. Aussi dégoûtés par les horreurs de leur époque que par l'inéluctable déliquescence de l'humanité, Céline et Drieu sont obsédés par les moyens de contrer ce que l'historien Michel Winock appelle ironiquement l'« éternelle décadence ${ }^{5} »$ de la France. Selon eux, les descendants des colons français ont conservé les meilleurs éléments de l'Ancien Régime, qui vont 
de la vigueur physique à la piété, en passant par la ténacité campagnarde et la virilité, caractéristiques qu'ils ont pu conserver grâce au peu d'impact des idéaux de la Révolution française au Canada. Par exemple, Céline vouait une affection particulière à la mentalité présente tant dans les milieux scolarisés que dans les campagnes du Québec, cette province "où les curés sont rois ${ }^{6}$ ", parce qu'elle semblait prendre assise sur un mode de pensée similaire à ceux de la Bretagne et de la Normandie. En somme, le Canada français constituait pour Céline une sorte de "chouannerie transatlantique ${ }^{7}$ ». Quant à Drieu, il avoue admirer dans son roman Gilles la force brute de « ces braves Canadiens », de qui les Français devraient s'inspirer pour contrer l'insidieux poison de la décadence. Dans cette œuvre autobiographique ponctuée de dissonances idéologiques (antisémitisme, antiféminisme, fascisme), le mentor du héros, un historien des religions normand dont l'interprétation du christianisme se situe à la limite de l'hérésie, part en «mission de propagande au Canada ${ }^{8}$ » et en revient porteur de ce verdict:

il y a encore des Français, des êtres de chair et de sang, et d'âme, qui ne sont pas faits uniquement de livres et de journaux. C'est au nom de ces Français-là que je viens vous appeler... À part ça, c'est drôle de voir des Français, sur qui n'est pas passé 1789, ni le XVIII', ni même somme toute le XVII ${ }^{\mathrm{e}}$, ni même la Renaissance et la Réforme, c'est du Français tout cru, tout vif'.

Si elles ne reposaient pas sur un fonds véridique, ces remarques sur la rémanence de l'esprit prérévolutionnaire en Amérique du Nord nous feraient évidemment sourire. Or, nous savons que l'abbé Groulx tirait sa principale source d'inspiration stylistique du siècle de Bossuet, nous connaissons l'influence de l'ordre ecclésiastique au sein de l'appareil politique provincial d'avant 1960, ainsi que les craintes liées à la 
menace du communisme, concrétisation récente d'une volonté révolutionnaire $^{10}$. Mais au-delà de ces faits connus de tous, un entrefilet anodin de l'édition du 7 mai 1938 du quotidien Le Canada, rédigé à l'occasion du passage de Céline à Montréal, a aiguisé notre curiosité. On y parle entre autres de "l'écrivain israélite [sic] Jean-Richard Bloch», le désignant comme « un ennemi littéraire » de Céline, avant de conclure en ces termes: "[Céline] a autographié nombre d'exemplaires de son dernier roman [sic] Bagatelles pour un massacre ${ }^{11}$ ». Comment fut-il possible au journaliste (resté anonyme, d'ailleurs) de confondre un pamphlet antisémite avec un roman autrement que par un filtrage volontaire de l'information, surtout si nous considérons le fait que ce brûlot avait paru cinq mois plus tôt à Paris, soit en décembre $1937^{12}$ ? De plus, comment ignorer la frénésie populaire que ces Bagatelles semblent provoquer? Le journaliste a-t-il seulement pris connaissance de ce texte avant d'en parler aussi légèrement? Bref, s'agit-il d'une manifestation de l'esprit chouan dont parlent Céline et Drieu, ou alors d'une simple preuve d'ignorance, d'insouciance?

Afin de résoudre cette énigme, nous projetions à l'origine d'examiner de près la réception des œuvres de Céline et de Drieu par la critique québécoise des années 1930, soit dans La Relève, Les Idées, En avant!, Les Pamphlets de Valdombre, La Gazette littéraire, L'Ordre nouveau, La Revue moderne, L'Action nationale et L'Ordre. Nous espérions saisir l'idée maîtresse qui se dégageait de l'interprétation des deux premiers romans de Céline (Voyage au bout de la nuit et Mort à crédit), ainsi que des textes de plus en plus "engagés » de Drieu, de La Comédie de Charleroi à Gilles. En analysant l'accueil réservé à ces œuvres par les critiques québécois des années 1930, nous comptions démontrer que les idéologies de droite de la France et du Québec s'interpellaient et, en conséquence, que cet esprit 
chouan pressenti par Céline et Drieu ne relevait pas de la fantasmagorie. Or, après dépouillement du corpus, nous avons trouvé un maigre échantillon d'articles vagues, dont le ton allusif n'a fait que piquer davantage notre curiosité. Certes, le silence dévorant de la critique nous a forcé d'aborder le sujet autrement, mais l'essence de l'hypothèse demeure. En fait, l'esprit chouan remarqué chez les intellectuels québécois, en particulier chez les critiques, se manifeste dans l'orientation de leurs préférences littéraires, presque inévitablement tournées vers une esthétique conventionnelle, ainsi que dans le rejet systématique des tentatives trop hardies de renouveler la littérature. En somme, nous croyons que la discrétion de la critique tient à deux facteurs majeurs : elle est d'abord imputable à la tonalité avant-gardiste des premiers textes de Drieu et de Céline (avec tous les écarts normatifs que prône cette esthétique), mais aussi à l'idéologie sous-tendue par leurs œuvres (d'orientation floue, mais assurément subversive). Après une brève analyse des rares articles dans lesquels ils sont mentionnés, nous traiterons du rôle de la critique et de son attitude réfractaire vis-à-vis des inventions verbales de l'avant-garde et des œuvres sympathiques à une cause subversive. À défaut de savoir avec exactitude ce qu'on pensait de Céline et de Drieu, nous verrons à tout le moins que, dans un contexte culturel où la critique constructive n'était pas absolument exclue, le quasi-silence à l'égard de certains auteurs qui haussaient anormalement la voix révèle, un peu à la manière du négatif photographique, des détails d'une importance insoupçonnée.

\section{L.-F. Céline (1894-1961) et P. Drieu la Rochelle (1893- 1945)}

À défaut d'avoir emprunté des parcours esthétiques et idéologiques similaires, André Breton, Louis Aragon, Paul 
Éluard, Emmanuel Berl, Jean Giono et Henry de Montherlant appartiennent à la même génération que Céline et Drieu. Qu'ils aient participé aux manœuvres du front dès le déclenchement des hostilités (comme Céline et Drieu) ou qu'ils se soient contentés à cause de leur trop jeune âge de pleurer la perte de leurs camarades dans l'hécatombe (comme Sartre et Malraux), tous les écrivains de cette génération partagent d'une manière ou d'une autre le traumatisme de la Première Guerre : "On est puceau de l'Horreur comme on l'est de la volupté ", écrit Céline dans son célèbre Voyage au bout de la nuit. "Comment aurais-je pu me douter moi de cette horreur en quittant la place Clichy ${ }^{13}$ ?». Avant qu'il ne devienne l'écrivain controversé que nous connaissons aujourd'hui, l'auteur du Voyage n'était connu que sous le nom de Louis-Ferdinand Destouches, docteur de la Faculté de médecine de Paris. Parallèlement à son poste de médecin au dispensaire de Clichy, banlieue populaire située au nord-ouest de Paris, Destouches participa jusqu'en 1933, à titre d'hygiéniste officiel de la Société des Nations, à plusieurs tournées d'inspection et d'information en Europe et en Amérique ${ }^{14}$. Rescapé de ce que plusieurs ont baptisé «la grande boucherie », réformé en raison de ses graves blessures et, enfin, médecin des pauvres, Céline s'affirme par le truchement de son personnage Bardamu comme un farouche défenseur de la paix à tout prix et un critique du capitalisme. François Gibault commente l'enthousiasme soulevé par Voyage chez les intellectuels de la gauche révolutionnaire et modérée, qui virent d'abord en Céline un compagnon d'armes potentiel :

Il n'est pas douteux qu'Aragon et Elsa Triolet ont aimé Voyage, comme beaucoup de gens de gauche, notamment parmi les communistes. Le livre était pacifiste et anticolonialiste, un peu anarchiste et donnait une image assez hideuse de la société bourgeoise. Aussi beaucoup de communistes ont-ils vu en Céline un écrivain prolé- 
tarien. Georges Altman dans Monde et Paul Nizan dans L'Humanité avaient été séduits par la vision célinienne d'une société capitaliste en pleine décomposition ${ }^{15}$.

Mais cet engouement pour Céline et sa révolution stylistique ne dura qu'un moment, c'est-à-dire jusqu'à la parution en 1936 de Mort à crédit, en quelque sorte la suite du Voyage. En poussant sa recherche du renouvellement de la langue romanesque dans les derniers retranchements du registre argotique et en invectivant tout le monde, surtout les petites gens, Céline ne plut à personne. Gibault rapporte qu'il «fut attaqué de partout. L'éreintement vint autant de la gauche que de la droite ; il portait à la fois sur le fond du livre et sur sa forme [...]. Pour la droite, le livre était décadent, obscène et scatologique. À gauche, on y trouvait curieusement un arrière-goût fasciste $^{16} »$. Têtu, anticonformiste et irrévérencieux, Céline ne chercha pas une seconde à regagner la confiance de ses premiers admirateurs. Au lieu de renouer avec le ton du Voyage pour calmer les esprits, Céline répliqua avec de redoutables pamphlets d'humeur - Mea Culpa (1936), Bagatelles pour un massacre (1937), L'École des cadavres (1938) et Les Beaux Draps (1941) - dans lesquels l'antisémitisme, l'anticommunisme, les idées fascisantes et le nihilisme pur se côtoient. Les propos délirants tenus par Céline dans ces quatre brûlots successifs le contraignirent à l'exil au lendemain de la Libération, d'abord en Allemagne en compagnie du gouvernement de Vichy en exil, puis au Danemark jusqu'à ce qu'il fût amnistié en $1951^{17}$. Étrangement, partout en Occident, sauf au Québec, l'extrémisme des vaticinations proférées par Céline ainsi que son style novateur suscitèrent dans la presse un flot continu de commentaires. En effet, malgré certaines allusions furtives à d'autres titres ou à la polémique soulevée à Paris par la parution de Bagatelles ${ }^{18}$, il semble demeurer tout au long des années 1930 l'auteur du seul Voyage. 
Contrairement à celle de Céline, la carrière littéraire de Drieu s'amorça très tôt. Avant même la trentaine, il publia deux recueils de poèmes aux échos claudéliens inspirés de sa douloureuse expérience au front lors de la Première Guerre mondiale, soit Interrogation (1917) et Fond de cantine (1920). Touché à la tête dès les premières charges à Charleroi, blessé de nouveau en Champagne, évacué des Dardanelles pour dysenterie, puis blessé à Douaumont après la bataille de Verdun, Drieu garda de la guerre, la plus grande désillusion de sa jeunesse, un souvenir indélébile inscrit dans sa chair même : " [C]'était une carcasse foudroyée, tourmentée, tordue, desséchée, chétive, dira-t-il du côté gauche de son corps dans Gilles. C'était le côté de la guerre, du massacre, du supplice, de la $\operatorname{mort}^{19} \gg$. Au début des années 1920, encore dégoûté par les manœuvres « antidiplomatiques ${ }^{20}$ » responsables de cette guerre inutile et obsédé par l'idée de la décadence européenne, Drieu fréquenta Breton, Aragon, Soupault et Éluard, principaux acteurs du groupe surréaliste et de la revue Littérature, partageant avec eux le nihilisme d'une génération confrontée au vide politique et moral de l'entre-deuxguerres. Aussi l'apolitisme initial du mouvement ainsi que l'idée d'une révolution sociale par le renouvellement systématique de l'art exercèrent-ils sur lui quelque influence - l'essai $M e$ sure de la France (1922), les nouvelles de Plainte contre inconnu (1924) et le roman L'Homme cowvert de femmes (1925) sont les fruits de cette période. Toutefois, cette fascination sera rompue en même temps que son amitié pour Aragon par l'entremise d'une lettre ouverte publiée dans la Nouvelle Revue Française en 1925, dans laquelle il dénonce les prises de position arbitraires «entre le zist et les zest ${ }^{21} »$ des surréalistes, notamment leur engagement hâtif pour le communisme. Après cette rupture, toujours écartelé entre les idéologies de droite et de gauche, entre la nation et l'Europe, entre son moi et la collec- 
tivité, Drieu poursuivit ses réflexions entre des séjours à Berlin, Moscou et Buenos Aires, tâtant des formes littéraires les plus diverses: articles politiques et littéraires, essais, romans, nouvelles et pièces de théâtre. Mais si l'on en croit l'épisode rapporté dans Gilles, ses louvoiements politiques et philosophiques prirent véritablement fin le 6 février 1934, à l'occasion des émeutes de la Place de la Concorde fomentées par l'Action française, les Croix-de-Feu et autres organisations d'extrême droite. Ainsi, après avoir été le plus discipliné des écrivains associés de près ou de loin à l'avant-garde, après avoir accusé Aragon de tomber dans la facilité de l'engagement politique, Drieu publie cette année-là un recueil d'articles intitulé Socialisme fasciste, dans lequel il trahit son inclination pour le kitsch constitutif du fascisme, utopie politique des apparences ${ }^{22}$. Ajoutée aux thèmes jusque-là exploités par Drieu (la vacuité de la séduction et de l'érotisme, l'amour impossible, l'inéluctabilité de la décadence et de la mort, bref la déréliction), cette prise de position clairement subversive et antihumaniste ne pouvait contribuer à rendre sa signature moins louche auprès des intellectuels québécois, ni favoriser la popularité de ses œuvres dans les librairies. Enfin, à la Libération, en raison de sa collaboration trop étroite avec l'occupant, Drieu céda à la pulsion de mort qui le torturait depuis les tranchées de Charleroi et, après quelques tentatives manquées, se donna la mort chez lui le 15 mars $1945^{23}$.

\section{Céline et Drieu dans les périodiques québécois : quelques occurrences ${ }^{24}$}

En vérité, outre les textes signalant la visite de Céline à Montréal en mai 1938 (dans La Presse, Le Devoir, Le Canada et En avant .), nous n'avons pas réussi à trouver un seul développement substantiel portant sur l'un ou l'autre des deux auteurs. Pourtant, la publication du Voyage au bout de la nuit en 
1932, et surtout la polémique occasionnée par l'attribution du prix Goncourt à Guy Mazeline (alors que la critique parisienne et même certains membres du jury, dont Léon Daudet, le destinaient à Céline), a suscité une avalanche d'articles et de comptes rendus dans la presse française. Dans la seule année suivant l'«affaire Goncourt», soit de novembre 1932 à décembre 1933, on a dénombré pas moins de cent soixantedix articles consacrés au Voyage, non seulement en France, mais aussi en Belgique, en Suisse, aux Pays-Bas, en Italie, au Luxembourg et aux États-Unis ${ }^{25}$. D'ailleurs, les critiques littéraires français les plus renommés, notamment Léon Daudet, Edmond Jaloux et Ramon Fernandez, rendirent compte de la révolution stylistique célinienne dans des revues aux orientations idéologiques aussi variées que la Nouvelle Revue Française, Marianne, Candide, Comodia, Esprit, La Revue des deux mondes, Les Nouvelles littéraires, etc. Dans un article publié dans Candide, le 22 décembre 1932, Daudet salua les accents retentissants du roman de Céline, qu'il considérait comme la version «mâle " de l'auto-analyse proustienne étendue à la décrépitude planétaire ${ }^{26}$. De toute évidence, malgré le cautionnement de l'œuvre par Daudet, qui jouissait en tant que fondateur de l'Action française d'un grand prestige dans les milieux nationalistes canadiens-français, nos intellectuels décidèrent de ne pas emboîter le pas à leurs collègues, et ce bien qu'ils eussent à tout le moins pris connaissance de la tempête par le biais des revues parisiennes ${ }^{27}$. Cela ne signifie pas pour autant qu'on évite systématiquement de mentionner le nom de Céline, mais il faut avouer que les textes où il apparaît offrent bien peu à se mettre sous la dent. Certes, La Gazette littéraire mentionne bien Voyage au bout de la nuit (prix Renaudot 1932) dans son numéro de février 1934, le qualifiant fort justement de roman "désespéré, exaspérée ${ }^{28}$ », mais il semble que cette manœuvre vise uniquement à souligner le contraste 
avec l'optimisme du lauréat de 1933, Le Roi dort de Charles Braibant. Trois ans plus tard, l'hebdomadaire En avant! consacre un entrefilet amusant à l'aventure de l'écrivain à la poursuite de ses droits d'auteur en URSS. Davantage une pointe contre le régime de Moscou qu'un texte d'actualité littéraire, l'article intitulé «Il faut dépenser sur place » commence pourtant par cette question énigmatique: "Qui n'a pas lu le fameux Voyage au bout de la nuit de Céline qui est presque un voyage autour du monde moral et des misères humaines?", pour se terminer par une allusion à la parution prochaine de Mea culpa, un pamphlet anticommuniste ${ }^{29}$. Compte tenu du postulat sous-tendu par cette question (à moins qu'elle ne fût inspirée par la presse française, donc qu'elle ne fût que mimétique et purement rhétorique), nous serions en droit de nous demander qui, dans le Québec des années 1930, a vraiment lu Céline, pourtant "l'immortel écrivain du Voyage au bout de la nuit ${ }^{30}$ ", et pourquoi son nom se voit marqué, avant même sa série de pamphlets antisémites, d'une aura d'interdiction.

Quant au nom de Drieu, il apparait à quelques reprises, mais plutôt de manière accidentelle. Par exemple, le 7 mai 1937, un chroniqueur d'En avant! (encore la Main de velours) commente un article assez léger de Louis Martin-Chauffier publié dans l'hebdomadaire parisien Vendredi qui, selon Herbert Lottman, constitue " []a contribution la plus originale des écrivains à l'esprit du Front populaire ${ }^{31} »$. Drieu apparait ainsi aux côtés de Charles Du Bos, essayiste littéraire, Jean Guéhenno, l'un des rédacteurs de Vendredi et communiste affiché, Paul Claudel, poète et dramaturge catholique, et Jules Romains, auteur du roman-fleuve Les Hommes de bonne volonté, en sa qualité de... fumeur de pipe (alors qu'il fume plutôt des cigarettes)! Toujours dans En avant!, le 11 juin 1937, le même chroniqueur rend compte d'une enquête publiée dans les Nouvelles littéraires, influent hebdomadaire parisien situé « [a]u-des- 
sus des partis ${ }^{32}$ ", dans laquelle il était demandé aux écrivains : "Quel est le livre d'aujourd'hui que vous voudriez avoir écrit ?". La Main de velours nous apprend que "les contemporains les plus célèbres ont bien voulu répondre directement à la question » et que "Drieu La Rochelle [sic], si intelligent et si poli n'hésite pas à donner sa préférence ${ }^{33} »$. S’il disposait de l'opinion de contemporains célèbres, pourquoi donc a-t-il choisi d'énumérer les choix de Drieu la Rochelle (auquel d'ailleurs l'adjectif poli ne convient pas du tout), un écrivain qui semble pourtant inconnu auprès des intellectuels québécois et dont l'opinion ne devait intéresser qu'une minorité $e^{34}$ ? Certes, Drieu avoue être « fasciné par les livres mystiques » et admirer Bernanos, Mauriac, Psichari, Bloy et Claudel, auteurs pour la plupart prisés par les élites québécoises, mais ses œuvres de fiction, en plus de contenir de nombreuses scènes sensuelles osées, n'affichent pas la moindre foi ni élévation spirituelle.

Néanmoins, le peu d'écho engendré par les romans et les nouvelles de Drieu ne nous permet pas d'affirmer qu'il fût totalement inconnu au Québec. Car à défaut de fréquenter l'écrivain, les intellectuels québécois qui s'abreuvaient aux revues politiques et littéraires parisiennes ${ }^{35}$ le connaissaient en tant que journaliste. En effet, dès les années 1920, Drieu collabora à plusieurs revues, dont la Nouvelle Revue Française (avant d'en assumer la direction sous l'Occupation), Marianne, Comodia, Les Nouvelles littéraires, ainsi que le Figaro, Candide, Je suis partout et Gringoire, toutes régulièrement citées dans les périodiques du Québec de l'entre-deux-guerres. Nous avons même trouvé deux articles de Drieu reproduits in extenso. Le premier, publié à l'origine dans Marianne, « [o]rgane illustré de la gauche modérée, temporairement lié au Front populaire ${ }^{36}$ » dirigé par son ami Emmanuel Berl, écrivain "politiquement neutre $^{37}$ ", fut repris par La Gazette littéraire. Drieu y rendait 
compte assez cyniquement de deux livres portant sur les émeutes politiques de février 1934, en ridiculisant à mots couverts les discours de la droite et de la gauche. Sa critique de La Journée du 6 février de Georges Imann et des Journées ouvrières des 9 et 12 février de Marc Bernard se clôt sur ce verdict aussi flou qu'assassin : "Pour le moment, comme le montrent ces deux livres, nous sommes entre des gens de droite qui parlent de députés corrompus en oubliant les banquiers et industriels corrupteurs, et des gens de gauche qui croient que la révolution sociale, c'est de défendre son bureau de $\operatorname{tabac}^{38} »$. Le second article de Drieu, d'abord publié dans le journal conservateur Le Figaro, fut reproduit dans L'Ordre du 3 février 1935 ; prenant pour appui les exemples de l'Allemagne et de l'Italie, deux dictatures dont « le programme social n'est pas du tout féministe ${ }^{39}$ ", Drieu soutient que l'égalité juridique entre les sexes ne découlera pas automatiquement de l'inscription du droit de vote des femmes dans la constitution française. Bien que sommaire et franchement plus désabusée que conservatrice (Drieu laisse entendre que la démocratie a failli à la tâche), son analyse a tout pour conforter l'opinion et les certitudes de ceux qui, encore en 1935, s'opposaient au droit de vote des femmes dans la province de Québec.

\section{Le rôle de la critique littéraire des années 1930 : l'avant-garde en horreur}

"Je me déclare mondial, ovipare, girafe, altéré, sinophobe et hémisphérique. Je m'abreuve aux sources de l'atmosphère qui rit concentriquement et pète de mon inaptitude ». Ces lignes, extraites du Cornet à dés, de M. Max Jacob, sont une œuvre d'art, un poème en prose, un bijou. C'est du moins l'auteur qui l'écrit dans sa préface ${ }^{40}$. 
Ainsi, ni les critiques ni les chroniqueurs littéraires n'ont osé parler directement de Céline ou de Drieu, et ce bien que leurs œuvres aient suscité de nombreuses recensions dans les revues parisiennes prisées ici. Nous pouvons supposer que les préférences et les intérêts des intellectuels québécois se trouvaient ailleurs : si la génération de La Relève considérait la revue Esprit de Maritain et Mounier comme une nourriture spirituelle, d'autres intellectuels furent marqués par le style réactionnaire de l'Action française. Cela ne destinait aucun de ces deux groupes à se délecter en public des propos d'abord antinationalistes (Mesure de la France, qui déplut fortement à Maurras, et L'Europe contre les patries), puis fascistes (Socialisme fasciste), mais surtout sensualistes et cyniques de Drieu, ni des romans noirs et anticonformistes de Céline ${ }^{41}$. En fait, il n'est pas nécessaire de fouiller bien loin pour dresser un panorama représentatif des auteurs français qui retenaient l'attention des critiques du Québec et tombaient dans leurs bonnes grâces. Outre les classiques expurgés présentés dans les collèges classiques, on affiche un faible pour le XIX ${ }^{e}$ siècle (sans tout à fait pardonner certains écarts normatifs des romantiques, puis de Balzac, Flaubert et Zola), on lit avec plaisir Paul Bourget, Henry Bordeaux, Julien Green, Paul Claudel, Léon Daudet et Maurice Barrès, on se risque parfois dans André Gide, Henry de Montherlant, François Mauriac, Georges Duhamel et Paul Morand. D'ailleurs, même le dépouillement exhaustif de quelques-uns des périodiques mentionnés plus tôt n'a donné lieu qu'à fort peu de surprises, la plupart d'entre elles amusantes et, surtout, très révélatrices de l'extrême conservatisme littéraire qui régnait (officiellement, du moins) dans les sphères intellectuelles du Québec de l'entre-deux-guerres.

Évidemment, ce jugement sévère porté à rebours eût trouvé à l'époque maints détracteurs parmi ceux qui se piquaient d'être au courant des plus récentes publications, des 
dernières actualités parisiennes. Mais compte tenu de la masse documentaire témoignant de la popularité d'un noyau de romanciers français, cette affirmation s'avère assez facile à étayer. Par exemple, dans un article paru dans L'Action nationale, Harry Bernard se félicite de l'ouverture d'esprit perceptible chez les intellectuels québécois, auxquels les contemporains n'inspirent plus la peur : «Le temps n'est plus où Radiguet était chez nous une sorte d'épouvantail, où les noms de Mauriac et de Morand, de Maurois, de Claudel ou de Jules Romains provoquaient l'hilarité, quand ce n'était pas de sceptiques haussements d'épaules ${ }^{42} »$. Malgré ce qu'en pense Bernard, les critiques restent généralement timorés. Si la seule mention du nom de Claudel, bientôt adulé par toute la droite catholique, fut sujette à provoquer le rire, nous pouvons nous demander ce qui arrivait lorsque surgissait celui d'un esprit contestataire ou d'un représentant de l'avant-garde !

Grâce à quelques indices épars, nous savons que des œuvres littéraires d'avant-garde, d'inspiration dadaïste ou surréaliste, circulaient dans les milieux cultivés du Québec des années 1930, sans toutefois causer chez les critiques la plus inoubliable des expériences esthétiques. Parmi ceux ayant le plus régulièrement collaboré aux revues et hebdomadaires québécois des années 1930, Berthelot Brunet et Claude-Henri Grignon, alias Valdombre, nous ont semblé les plus loquaces. À quelques exceptions près, ce sont eux qui offrent les articles aux vues les plus surprenantes, tout en demeurant assez conventionnels dans leurs jugements pour servir de baromètre des goûts littéraires officiels de l'époque. Victor Barbeau disait de Brunet que " [s]es papiers mal pensés, mal ficelés ne servaient guère les lettres canadiennes » et que «[m]alingre, souffreteux, il écrivait une langue flasque, gélatineuse, qui ne rendait pas justice à son érudition ${ }^{43} »$. Certes, contrairement au style affecté de Barbeau, celui de Brunet ne scintille pas 
jusque dans l'adjectif le plus retranché. En revanche, l'éclectisme de sa culture livresque éclate dans chacune de ses phrases tortueuses. Évidemment, Brunet a fréquenté les écrivains français d'après-guerre, dont plusieurs étaient des fils spirituels de Maurice Barrès, mais il ressent la nécessité de prendre ses distances : «j'ai laissé tomber il y a belle lurette Kessel, Carco, Pierre Benoit, Dorgelès, Chérau, Giraudoux, Delteil, Green, Montherlant, Durtain, Mauriac, en dépit de trois ou quatre bons ouvrages. Maurois, malgré Disraeli et Édonard VII. Ils vont rejoindre dans mon cimetière littéraire les Bourget, les Bordeaux, les Prévost, qui eux aussi ne manquaient pas de talent ${ }^{44} »$. Comparativement à ses collègues, Brunet présente une remarquable ouverture d'esprit qui lui fait lire Proust, qu'il considère tout de même comme « un homme du monde Terrible ${ }^{45}$ ", l'Américain John Dos Passos et le romancier anglais D. H. Lawrence, ainsi que les dernières traductions française et anglaise des Autrichiens Joseph Roth et Arthur Schnitzler. Brunet n'a peut-être pas perçu la beauté ni l'importance de La Marche de Radetzky de Roth, chronique du déclin de la famille von Trotta, le trouvant «d'une lourdeur extrême, et d'une vulgarité fort médiocre ", mais il voue en revanche une grande admiration à Schnitzler, peintre par excellence de la décadence fin de siècle de Vienne : «C'est fait de rien, et ce sont deux tours de force. L'auteur a laissé le récit pour le monologue intérieur, ce fameux monologue intérieur qui rendit célèbre James Joyce et auquel Valéry Larbaud s'est vainement essayé en France ${ }^{46} »$. Le seul fait d'admirer des auteurs comme Schnitzler, qui fit scandale en raison notamment de la crudité de sa pièce La Ronde ${ }^{47}$, et de citer judicieusement Joyce, dont le roman Ulysse fut accusé d'obscénité par la presse anglo-saxonne avant d'être censuré, montre l'inclination de Brunet pour la modernité littéraire et sa relative indépendance d'esprit vis-à-vis de ses collègues. Malgré 
certains relents de pudibonderie au sujet de L'Amant de Lady Chatterley, pour lequel D. H. Lawrence aurait mérité «la camisole de force ${ }^{48}$ ", Brunet demeure un critique fort original quant aux livres et aux sujets abordés, ainsi que l'un des plus habiles pour étaler son érudition. Néanmoins, jamais il ne mentionne Céline, Drieu ou l'avant-garde parisienne, pas même pour en dire du mal.

Une autre figure importante du champ intellectuel québécois s'est toutefois prononcée librement sur la question de l'avant-garde littéraire. Contrairement à Brunet, dont il fut en quelque sorte le mentor, Claude-Henri Grignon propose surtout, dans En avant! et Les Pamphlets de Valdombre, des analyses brèves rédigées sur un ton approprié au titre de sa revue. S’ils sont appréciés, les nouveaux livres reçoivent de sa part un traitement exemplaire et la justesse de ses observations s'avère troublante. Par exemple, il a tout de suite saisi la profondeur du renouveau esthétique impliqué par Regards et jeux dans l'espace de Saint-Denys Garneau, ainsi que l'importance historique du Retour de l'URSS de Gide. Par contre, si par malheur les nouveautés ne lui plaisent pas (cela arrive très souvent), Valdombre quitte alors les sentiers de la clairvoyance pour tomber dans l'aveuglement réactionnaire le plus commun. Cette attitude se manifeste de manière assez évidente dans la section littéraire de l'hebdomadaire En avant!, page dont Valdombre assume la direction et pour laquelle il rédige la plupart des textes. En plus de présenter les actualités du monde littéraire français et québécois et de publier des articles de fond, il réserve régulièrement un espace à la reproduction d'un poème contemporain, tantôt pour en admirer les qualités esthétiques, tantôt pour le démolir sans retenue ni le moindre discernement. S'il est vrai qu'il se livre assez rarement à ce genre d'exercice, lequel s'apparente souvent à l'attaque ad hominem, chaque occasion dont il profite donne lieu 
à des observations d'une rare densité idéologique. Le procédé rhétorique qu'il emploie s'avère plutôt simple : il présente aux lecteurs les poèmes avant-gardistes les plus obscurément complexes et justifie leur publication dans En avant! en prétextant leur «beauté toute moderne » tout en s'excusant de céder à cette mode ridicule.

Au cours de l'existence d'En avant!, Valdombre s'est d'abord attaqué à Tristan Tzara, initiateur et figure centrale du mouvement Dada. Fondé à Zurich en 1916 par Tzara et Hugo Ball, ce groupe nihiliste et subversif prônait notamment la destruction de toutes les normes esthétiques établies, celles de l'art comme celles du langage ${ }^{49}$. Dans la première Aventure céleste de M. Antipyrine (1916), poème-manifeste qui inaugura une série de déclamations spectaculaires et dont Valdombre ampute le titre, Tzara énonce bien le programme du mouvement : «Dada est l'art sans pantoufles ni parallèle; qui est contre et pour l'unité et décidément contre le futur ; nous savons sagement que nos cerveaux deviendront des coussins douillets que notre anti-dogmatisme est aussi exclusiviste que le fonctionnaire que nous ne sommes pas libres et que nous crions liberté Nécessité sévère sans discipline ni morale et crachons sur l'humanité ${ }^{50} »$. C'est imprégné de cet esprit de révolte radical que Tzara écrivit les vers que Valdombre se garda bien de reproduire dans leur intégralité, multipliant les altérations et les oublis:

il y a une grande auréole où les vers circulent en silence car les vers et les autres animaux ont aussi des peines des douleurs des inspirations regarde l[es] fenêtre[s] qui s'enroule[nt] comme des girafes tournent se multiplient hexagones grimpent tortues la lune se gonfle marsupial et devient chien [lara et le cacatoès admirent le chien] un lys vient d'éclore dans le trou de son [cul] ${ }^{51}$ 
Le ton employé par Valdombre dans son commentaire trahit à la fois son faible intérêt pour la question de l'avantgarde et son ignorance du sujet. À défaut d'être une « grande œuvre littéraire » au sens canonique du terme, le poème de Tzara a le mérite d'exprimer formellement, par une distorsion du sens et de la syntaxe, la révolte sociale projetée par Dada. Selon Henri Béhar, "chez lui, la destruction est créatrice. Il fait table rase du passé et construit sur nouveaux frais en acceptant ses propres contradictions, en ouvrant les écluses de sa pensée. Dès lors, on assiste à un extraordinaire foisonnement d'images, qu'aucune règle logique n'aurait permis de découvrir $^{52} »$. Toutefois, au lieu de replacer le poème de Tzara dans son contexte de création, Valdombre l'interprète au pied de la lettre, afin de ridiculiser à la fois son auteur et ceux qui le lisent:

On peut croire qu'on se moque ici de nos lecteurs. Pardon! ces beaux vers ont paru dans l'Anthologie $d u$ Surréalisme et je connais ici, dans le Québec, des grands esprits qui les connaissent par cœur et se font fort de les réciter à qui veut les entendre. On ne court qu'un danger: devenir subitement fou. À part cela, rien à craindre ${ }^{53}$.

En plus de dénaturer le poème en changeant son titre, en modifiant son organisation strophique et en y insérant des coquilles, pas une seconde il ne cherche à saisir l'esprit du projet camouflé derrière ces vers en apparence décousus : il se contente de mettre Tzara en joue et de tirer contre son texte une salve chargée de sarcasmes, un de ses "poisons distillés $^{54} »$. Ironie du sort, on considère aujourd'hui les pieds de nez de Tzara comme des textes fondateurs. Ils sont devenus (à rebours, sans doute) un point de référence dans la littérature occidentale du $\mathrm{XX}^{e}$ siècle, la faisant bifurquer vers des univers inexplorés. Quant à Grignon, malgré son esprit sa- 
gace, il ne subsiste que par son roman Un homme et son pérhé (dont la fortune est considérable, mais sur un territoire réduit) ou encore, comme nous venons de le voir, par le biais de notes réfractaires à l'avant-garde et imperméables à son humour noir.

Les attaques de Valdombre ne visent toutefois pas que les délires volontaires de Tzara ni les «poètes pas mal fous qui écrivent des idioties de cette qualité-lă ${ }^{55} »$. En fait, le contenu subversif des textes d'avant-garde cités, de même que certaines «maladresses " commises par les auteurs écorchés, peuvent expliquer sa condescendance. Mais après examen, force nous est d'avouer que ses railleries ne sont ni toujours justifiées ni très habiles, surtout lorsqu'il s'en prend (comme d'autres conservateurs de son époque, il est vrai) à des auteurs dont on a depuis reconnu l'importance. Le cas de Tzara nous a montré ce que Valdombre pouvait penser des audaces verbales excessives (les dadaïstes se sont surtout adonnés aux explorations poétiques autoréférentielles les plus échevelées), mais la charge du critique contre Paul Éluard s'explique plus difficilement. À l'époque où Valdombre reproduit son poème, Éluard avait déjà publié Capitale de la douleur (1926), L'Amour, la poésie (1929) et La Vie immédiate (1932), trois recueils nourris aux thèmes chers aux surréalistes : l'amour fou, la spontanéité et l'inconscient. Faussement intitulé «La Vie immédiate », soit le titre du recueil dont il est tiré, l'extrait du poème repris par Valdombre constitue en fait un hommage à Salvador Dalí. C'est d'ailleurs pour cette raison que la force de ses associations verbales rappelle les incongruités visuelles surréalistes du Chien andalou, manifeste filmique réalisé par Buñuel et Dalí en 1929. Afin de montrer le peu d'intérêt que présentait le poème d'Éluard aux yeux de Valdombre, nous le citons tel qu'il fut imprimé dans En avant!, c'est-à-dire avec ses coquilles et son organisation strophique approximative : 
C'est pour ne pas laisser ces yeux les nôtres vide[s] entre nous

Qu'elle tend ses bras nus

La fille sans bijoux la fille à la peau nue

Il faudrait bien par-ci par-là des roche[r]s des vagues

Des femmes pour nous distraire pour nous habiller

Ou des cerises d'émeraude dans le lait de la rosée.

Tant d'aubes brèves dans les mains

Tant de gestes maniaques pour dissiper

l'insomnie

Sous la rebondissante nuit du linge

Face à l'escalier dont chaque marche est le plateau d'une balance

Face aux oiseaux dressés contre les torrents

L'étoile lourde du beau temps s'ouvre les veines ${ }^{56}$.

Deux ans après avoir démoli Tzara, Valdombre adresse à Éluard et ses lecteurs du Québec cette note assassine, qu'on aimerait croire davantage inspirée par une impulsion passagère que par son intellect :

C'est dommage que nous ne puissions pas reproduire la première partie de cette "Vie Immédiate » [sic] qui vaut à elle seule toute la vie. Un si beau poème nous arrache les larmes en nous procurant un de ces maux de tête qu'on n'oublie plus. Il n'y a pas à dire, la poésie française n'est pas près de mourir ou prête à mourir. Quand on pense que des petits jeunes gens de chez nous s'imaginent que c'est là de la haute poésie. Que du haut de ses nuages le Père Éternel leur pardonne ${ }^{57}$ !

L'ironie et l'impertinence du commentaire, tout comme la rigidité esthétique qu'elle sous-tend, semblent aujourd'hui aussi inconvenants, voire davantage, que le refus poli et pu- 
sillanime adressé par la maison Gallimard à Proust et à Céline pour la publication de leur premier roman. Mais contrairement au plus célèbre des éditeurs parisiens, pour qui la longueur démesurée des romans de Proust et de Céline pouvait représenter un investissement trop risqué, Valdombre dirigeait sa propre revue, s'exprimait volontairement sur un mode aussi péremptoire que professoral et visait à faire autorité. Il est demeuré imperméable à la fraîcheur poétique du nouveau mouvement littéraire européen, allant même jusqu'à s'engager résolument contre pendant au moins deux ans. D'ailleurs, à force de lire les notes acidulées que Valdombre adressait avec un acharnement messianique aux poètes modernes après avoir cité hors contexte la partie la moins réussie de leur œuvre, nous nous demandons si le titre de l'hebdomadaire En avant! ne constituait pas un contresens : comment peut-on sérieusement prôner à la fois le progrès et l'immobilisme, même en littérature?

\section{$* * *$}

Que pouvons-nous déduire du surprenant silence de la critique, de cette "non-réception » des œuvres de Céline et de Drieu, sinon une certaine crainte de l'engagement ambigu ? Le fait qu'un intellectuel comme Victor Barbeau ait avoué sa préférence pour Céline bien après l'entre-deux-guerres nous porte à croire que l'inclination pour le style et les idées de ce non-conformiste (comme pour les écrits "décadents" de Drieu, dans une moindre mesure) était à l'époque inavouable. Ces auteurs étaient-ils trop virulents, voire trop à droite pour nos élites à tout le moins familières avec les éditoriaux de L'Action française? Ou alors trop cyniques, trop désabusés pour que leur discours fût reçu comme autre chose qu'un renoncement à la vie, à l'espoir de rédemption ? Bien qu'il soit impossible de répondre à ces questions de manière absolue, 
nous pouvons, à la lumière de la réception de la poésie française d'avant-garde par Valdombre, risquer quelques hypothèses. D'abord, en raison de la prégnance de l'idéologie conservatrice, en politique comme en littérature, parmi les élites intellectuelles du Québec des années 1930, nous pouvons supposer que plusieurs idées maîtresses des auteurs français de droite, dont l'obsession de la décadence morale présente chez Céline et Drieu, ont dû être récupérées et reformulées. Car s'il avait existé un désaccord fondamental entre Céline, Drieu et les intellectuels québécois, c'est-à-dire une indignation semblable à celle de Valdombre devant la poésie française d'avant-garde, la critique se serait sans aucun doute exprimée ouvertement contre eux sans retenue. En conséquence, le silence qui entoure l'œuvre de Céline et Drieu dans les périodiques québécois des années 1930 pourrait constituer un signe de prudence, voire de pudeur, de la part de la critique, une indifférence de façade. Cet état de fait n'implique nullement que les intellectuels québécois n'aient marqué aucun intérêt pour ces écrivains. Nul doute qu'ils se sont risqués à fréquenter ces auteurs "peu recommandables ", mais en raison de leur esprit subversif et anticlérical, du langage ordurier observable chez Céline ou encore de la large place accordée par Drieu au corps féminin, il nous est permis de croire qu'il leur était préférable de réserver leurs opinions favorables aux sphères privées qui les accueillaient, de se limiter à des propos de salons désengagés, pour éviter de s'exposer aux remontrances des autorités morales par un acte d'écriture indélébile.

\section{NOTES}

${ }^{1}$ Cet article est la version remaniée d'une communication présentée au Congrès de l'ACFAS le 16 mai 2000 à l'Université de Montréal, dans le cadre du colloque Les intellectuels et les années 1930 : un brassage idéologique, organisé par 
Sylvie Lacombe, Pierre Skilling et Frédéric Boily. Nous tenons à remercier tout spécialement Lise Couillard, Frédéric Gagnon et Frank Runcie pour leurs commentaires éclairés.

${ }^{2}$ Philippe Hamon. Texte et idéologie. Paris, Presses Universitaires de France, 1984. 227 p.

${ }^{3}$ Octave Aubry, « Le miracle canadien », L’Ordre, 24 janvier 1935, p. 2.

${ }^{+}$Henry Bordeaux, Nouvelle et vieille France, Paris, Plon, 1934, p. 96.

5 Michel Winock, Nationalisme, antisémitisme et fascisme en France, Paris, Éditions du Seuil, 1990, pp. 103 et suivantes. (Coll. " Points-Histoire »).

"Cité par Paul del Perugia, dans "Céline et le Québec ", Études littéraires, vol. XVIII, n 2 (automne 1985), p. 439.

${ }^{7}$ Ibid., p. 439.

${ }^{8}$ Pierre Drieu la Rochelle, Gilles, Paris, Gallimard, 1996 [1939/1942], p. 43. (Coll. « Folio »).

"Ibid., p. 161.

1" Compte tenu de l'attitude défensive adoptée par les intellectuels vis-à-vis du marxisme et de ses dérivés moins radicaux, il ne faut pas s'étonner de l'accueil plutôt froid réservé par la critique à des écrivains de gauche, comme Malraux par exemple, et ce malgré son engagement antifasciste. La citation suivante parle d'elle-même : «De ce qu'un homme a quelque don d'écriture, les idées qu'il peut avoir et les théories qu'il peut prêcher n'en sont ni meilleures ni pires. Il faut les voir telles qu'elles sont et lui tel qu'il est. Or Malraux est un grand-prêtre de Moscou, un ami de Staline, le Caucasien sanglant. [...] C'est donc un homme malfaisant. [...] Par conséquent, dire à Malraux et à ses pareils de nous ficher la paix, mettre l'opinion en garde contre eux, les montrer pour ce qu'ils sont (fauteurs de désordres, amis d'assassins, etc.), leur refuser la permission de prêcher ici la destruction, c'est faire œuvre nécessaire, salubre et bienfaisante - sans tenir compte des gloussements qui sortent de certains salons, où désaxées pernicieuses et « libérés » poseurs jouent avec la dynamite » (Louis Francœur, cité anonymement dans « Malraux à Montréal », L'Ordre nouveau, 20 avril 1937, p. 3.).

11 Anonyme. "Louis-Ferdinand Céline à Montréal ». Le Canada (Montréal), 7 mai 1938. Cité par Hélène Le Beau, dans « D’un voyage l'autre », Études littéraires, vol. XVIII, n 2 (automne 1985), p. 418. Les autres notes consacrées à la visite de Céline à Montréal sont parues le 7 mai 1938, dans Le Devoir et La Presse, puis le 20 mai 1938 dans En avant! 
${ }^{12}$ Voir « Sur les rives du Styx », chapitre que François Gibault consacre aux pamphlets et à l'antisémitisme délirants de Céline, dans Céline 1932-1944 : Délires et persécutions, Paris, Mercure de France, 1985, pp. 147-172.

${ }^{13}$ Louis-Ferdinand Céline, Voyage au bout de la nuit, Paris, Gallimard, 1994 [1932], p. 14. (Coll. « Folio »).

${ }^{14} \mathrm{C}$ 'est d'ailleurs au cours d'un de ces voyages qu'il entra pour la première fois en contact avec le Québec (Montréal, Trois-Rivières, Grand-Mère, Québec), soit en mai 1925. Voir Hélène Le Beau, loc. cit., pp. 417-432.

${ }^{15}$ François Gibault, op. cit., pp. 127-128.

${ }^{16}$ Ibid., pp. 118-119.

${ }^{17}$ Céline rapporte ce périple dans les romans qui composent sa trilogie allemande, soit D'un château l'autre, Nord et Rigodon, réunis dans Romans II, Paris, Gallimard, 1974. (Coll. «Bibliothèque de la Pléiade»).

${ }^{18}$ Voir Robert Élie, «Céline est resté un pessimiste invétéré », La Presse, 7 mai 1938.

${ }^{19}$ Pierre Drieu la Rochelle, Gilles, p. 501.

${ }^{20}$ Cet adjectif plutôt inattendu synthétise les perceptions de Drieu. À ce sujet, on peut consulter l'ouvrage de François Fejtö, Requiem pour un empire défunt. Histoire de la destruction de l'Autriche-Hongrie, Paris, Éditions du Seuil, 1993, pp. 23-59. (Coll. « Points-Histoire »).

${ }^{21}$ Pierre Drieu la Rochelle, «La véritable erreur des surréalistes », dans Sur les écrivains, Paris, Gallimard, 1964, p. 45.

${ }^{22}$ Nous nous référons ici à l'acception originelle du mot Kitsch, concept défini en ces termes par Hermann Broch, dans Création littéraire et connaissance, Paris, Gallimard, 1985 : « Le fait précisément de viser à la satisfaction des instincts par des moyens finis et rationnels, précisément cette pathétisation du fini jusqu'à la sphère de l'infini, ce travail tourné vers la réalisation de la "beauté", donnent au [kitsch] ce vernis d'insincérité derrière lequel on soupçonne le "mal" éthique. Car la fuite devant la mort qui n'est pas abolition de la mort, cette formalisation du monde qui cependant laisse le monde dépourvu de forme, ne sont aussi qu'une abolition illusoire du temps » (p. 364 ; traduction d'Albert Kohn, légèrement adaptée).

${ }^{23}$ Pour plus de détails sur Drieu et son œuvre, on consultera avec profit la biographie de Pierre Andreu et Frédéric J. Grover, (Drieu la Rochelle. Paris, La Table Ronde, 1989 [1979].) et l'ouvrage dirigé par Marc Dambre (Drieu la 
Rochelle, écrivain et intellectuel. Paris, Presses de la Sorbonne Nouvelle, 1995. 287 p.). Voir aussi Alice Y. Kaplan. «Bodies and Landscapes : Marinetti, Drieu la Rochelle, and Céline », dans Reproductions of Banality : Fascism, Literature, and French Intellectual Life. Minneapolis, University of Minnesota Press ("Theory and History of Literature », vol. 36), 1986, pp. 75-124 ; Heinz Thoma. « Nation - Nationalismus - Literatur: Gilles $(1939 / 1942)$ von Drieu la Rochelle ». Zeitschrift für Literaturwissenschaft und Linguistik, $\mathrm{n}^{\circ} 95$ (1994), pp. 79 90 et Susanne Rossbach. «Die Liebe als Kriegsersatz : Vorstellungen von Weiblichkeit im literarischen Werk Pierre Drieu la Rochelles », dans Joachim Born et Marion Steinbach, dir. Geistige Brandstifter und Kollaborateure. Dresde/ Munich, Dresden University Press, 1998, pp. 207-228.

${ }^{2+}$ Dans le cadre de cet article, dont les visées ne sont pas empiriques, nous avons concentré nos recherches sur les mensuels et les hebdomadaires, sans toutefois négliger les quotidiens. Nous avons d'abord systématiquement dépouillé les périodiques pourvus d'une section littéraire, soit $L a$ Gazette littéraire, L'Ordre, Les Pamphlets de Valdombre, L'Ordre nouveau, En avant!, L'Émérillon (de Québec, à ne pas confondre avec l'organe de l'Ordre de Jacques Cartier), L'Action nationale, La Relève et Les Idées, avant de jeter un coup d'œil au Devoir, à L a Presse, etc.

${ }^{25}$ Stanford L. Luce et William K. Buckley. A Half-Century of Céline. An Annotated Bibliography, 1932-1982. New York/Londres, Garland Publishing, 1983.

${ }^{26}$ Léon Daudet, cité par François Gibault, op. cit., p. 46. Gibault rapporte également que Daudet, nationaliste et antisémite notoire, chez qui on réservait toujours un couvert symbolique à Édouard Drumont, auteur de La France juive (p. 45), tenait le roman de Céline en si haute estime qu'il aurait dit à Paul Léautaud "La patrie, je lui dis merde, quand il s'agit de littérature » (p. 27).

${ }^{27}$ Bien qu'il commette une grave erreur factuelle en exprimant ses préférences littéraires, Victor Barbeau révèle bien l'opinion de l'élite intellectuelle des années 1930 : «D’avoir préféré Louis Pergaud à Colette, Henri Malherbe à Giraudoux, Thierry Sandre à Montherlant, Malraux [sic] à Céline refroidit singulièrement l'engouement que nous inspiraient les prix littéraires » (La Tentation du passé. Ressouvenirs, Montréal, Les Éditions La Presse, 1977, p. 81).

${ }^{28}$ G. Lavallée, La Gazette littéraire, février 1934, p. 3.

29 La Main de velours, En avant!, 5 mars 1937, p. 3. Les informations dont disposait le chroniqueur n'étaient certainement pas des plus récentes, car la 
publication de Mea culpa, bien qu'elle ne fit aucun bruit, « la gauche et la droite rivalisant d'indifférence à l'égard d'un texte dont personne ne mesura l'importance et qui dérangeait tout le monde » (François Gibault, op. cit., p. 151), remonte à la fin décembre 1936.

${ }^{30}$ En avant !, 20 mai 1938, p. 4 (note de la rédaction ajoutée à l'article que Laurence Talbot consacre à la visite de Céline à Montréal).

${ }^{31}$ Herbert R. Lottman, La Rive gauche, Paris, Éditions du Seuil, 1981, p. 133.

${ }^{32}$ Albrecht Betz, Exil et engagement. Les intellectuels allemands et la France 1933. 1940, traduit de l'allemand par Pierre Rusch, Paris, Gallimard, 1991, p. 305. (Coll. « Idées »).

${ }^{33}$ La Main de velours, En avant!, 11 juin 1937, p. 3.

${ }^{34}$ Pourtant, une liste publiée par La Gazette littéraire, mensuel littéraire et organe publicitaire de la Librairie Wilfrid Méthot, mentionne parmi les nouveaux arrivages du mois d'avril 1934 le plus récent livre de Drieu, La Comédie de Charleroi.

${ }^{35}$ En saluant les éphémères périodiques canadiens-français de l'entre-deuxguerres, Victor Barbeau remarque d'ailleurs ceci : « Sans elles les revues canadiennes-françaises], tout le champ eût été occupé par les périodiques de France. Il l'était déjà en majeure partie » (op. cit., p. 77).

${ }^{36}$ Albrecht Betz, op. cit., p. 305.

${ }^{37}$ Herbert R. Lottman, op. cit., p. 136.

${ }^{38}$ Pierre Drieu la Rochelle, « Livres sur février », La Gazette littéraire, mai 1934, p. 3.

${ }^{39}$ Pierre Drieu la Rochelle, «Quand les femmes votent... », L'Ordre, 3 février 1935, p. 4.

40 Anonyme, «Littérature moderne », L'Émérillon (Québec), mars 1936, p. 25.

${ }^{+1}$ Au sujet des revues québécoises des années 1930, voir la présentation qu'en fait Catherine Pomeyrols dans Les Intellectuels québécois: formation et engagements, 1919-1939, Paris/Montréal, L'Harmattan, 1996, pp. 232-248.

${ }^{42}$ Harry Bernard, « Histoire naturelle et Littérature », L'Action nationale, vol. I, $\mathrm{n}^{\circ} 1$ (janvier 1933), p. 21.

${ }^{43}$ Victor Barbeau, op. cit., p. 80.

${ }^{44}$ Berthelot Brunet, « Un “Art poétique” en 1934 », L'Ordre, 22 mai 1934, p. 4. 
${ }^{45}$ Berthelot Brunet, « Proust rue Sherbrooke », L'Ordre, 13 novembre 1934, p. 4.

t6 Berthelot Brunet, "J'en lis qui sont du Nord... », L'Ordre, 17 avril 1934, p. 4.

${ }^{47}$ Écrite en 1896/1897, cette pièce de Schnitzler fut représentée seulement à deux reprises du vivant de l'auteur, soit au moment de sa première à Berlin en 1920 et à Vienne en 1921. Au sujet de la pièce et du scandale, voir la préface de Günther Rühle, « Der ewige Reigen », dans Arthur Schnitzler, Reigen/Liebelei, Francfort, Fischer Taschenbuch Verlag, 1994, pp. 7-21.

${ }^{48}$ Berthelot Brunet, «J'en lis qui sont du Nord... », loc. cit., p. 4.

${ }^{49}$ Sur les accointances du mouvement $\mathrm{Dada}$ avec les milieux anarchistes, voir Hubert van den Berg. Avantgarde und Anarchismus. Dada in Zürich und Berlin. Heidelberg, Universitätsverlag Carl Winter ( Beiträge zur neueren Literaturgeschichte », 167). 1999.

${ }^{50}$ Tristan Tzara, La première aventure céleste de M. Antipyrine, dans CEuvres complètes I (1912-1924), Paris, Flammarion, 1975 [1916], p. 81.

${ }^{51}$ Tristan Tzara, «Aventure céleste » [sic], En avant!, 17 septembre 1937, p. 3. Nous rétablissons les béances du texte à partir de l'édition définitive des Cuvres complètes I (1912-1924) de Tzara, p. 83.

${ }^{52}$ Henri Béhar, "Introduction », dans Tristan Tzara, Dada est tatou. Tout est Dada, Paris, GF-Flammarion, 1996, p. 16.

${ }^{53}$ Valdombre [Claude-Henri Grignon], En avant!, 17 septembre 1937, p. 3.

${ }^{5+} \mathrm{Il}$ s'agit du titre d'une chronique régulière des Pamphlets de Valdombre.

${ }^{55}$ Valdombre [Claude-Henri Grignon], En avant!, 7 janvier 1938, p. 3. Il fait ici référence au poème d'un certain Silvaire, «Échecs », reproduit grâce à ses soins.

${ }^{56}$ Paul Éluard, [« Salvador Dalí »], En avant!, $1^{\text {er }}$ septembre 1939, p. 3. La version complète de ce poème tiré du recueil La Vie immédiate (1932) se trouve dans Paul Éluard, CEuvres complètes I, Paris, Gallimard, 1984 [1968], pp. 385 386. (Coll. «Bibliothèque de la Pléiade »).

${ }^{57}$ Valdombre [Claude-Henri Grignon], En avant!, $1^{\text {er }}$ septembre 1939, p. 3. 